Fernanda de Borbón and Daniel Ambrosini*

\title{
Influence of the nonlocal parameter on the transverse vibration of double-walled carbon nanotubes
}

DOI 10.1515/jmbm-2015-0010

\begin{abstract}
A high-order nonlocal continuum beam model is proposed, which can be applied to study the transverse vibrations of double-walled carbon nanotubes (DWCNTs), including those that could have initial deformations due to defects or external actions. A beam element is developed adopting Hermite cubic polynomials as shape functions, and mass and elastic stiffness matrix are presented. The influence of the nonlocal parameter on the vibrational properties of DWCNTs is studied. Using the proposed model, it was found that the nonlocal parameter has a strong influence on the natural frequencies.
\end{abstract}

Keywords: continuum beam nonlocal model; doublewalled carbon nanotubes; natural frequencies; vibration analysis.

\section{Introduction}

Due to the increasing importance of nanotechnology in the last years, carbon nanotubes (CNTs) have proven a great potential as a material because of their mechanical, thermic, and electrical superior behaviors in relation to other materials. A variety of applications are being studied such as nanoelectronics, nanodevices, liquid and gas carriers, and fillers in composite materials, among others [1-4].

There are three major approaches for studying CNT: atomistic simulations that include techniques such as molecular dynamics (MD) [5-7] with great precision but

*Corresponding author: Daniel Ambrosini, Structural Engineering Master Program, Engineering Faculty, National University of Cuyo, Centro Universitario, Parque Gral. San Martín, (5500) Mendoza, Argentina, e-mail: dambrosini@uncu.edu.ar; and National Scientific and Technical Research Council (CONICET)

Fernanda de Borbón: Structural Engineering Master Program, Engineering Faculty, National University of Cuyo, Centro Universitario, Parque Gral. San Martín, (5500) Mendoza, Argentina; and National Scientific and Technical Research Council (CONICET) high computational cost, hybrid atomistic-continuum models [8-10] where interatomic potentials are added through equating molecular potential energy with mechanical strain energy, and finally continuum models that simplify the structure to be considered but allow studying large systems with low computational cost. Continuum models can be developed as beam models, shell, or plate models [11-13].

The use of continuum models in small scales is questioned because of the discrete nature of CNTs and because, in nano- or microdimensions, the atomic lattice becomes important. In other words, the material properties are size dependent and the scale effect must be considered [14]. In the field of stress gradient elasticity theories, Eringen's nonlocal theory [15] states that the stress at a reference point depends on the strain field of all points in the body; therefore, information about interatomic forces should be added. Peddieson et al. [16] showed that nonlocal theory could be applied to nanotechnology. The major advantage of the nonlocal model depends on the scale parameter to be used, which must be properly adjusted. Eringen [15] defined the parameter $e_{0}$ like a constant appropriate to each material. However, the value of $e_{0}$ found in the literature differs with the nature of the problem and the model used for its calibration. Wang [17] pointed out that nonlocal theory should be used to correctly predict wave propagation, and he was the first to estimate the scale parameter. Duan et al. [18] calibrated the parameter for a Timoshenko beam model (TBM) and found that the parameter $e_{0}$ depends on the aspect ratio, vibration modes, and boundary conditions. Ansari et al. [19] validated a shell model with MD to obtain parameter values based on the boundary conditions showing that the values are not chirality dependent. Ansari and Sahmani [20] adopted a different nonlocal parameter corresponding to different nonlocal beam theories.

Various available beam theories, including the Euler-Bernoulli, Timoshenko, Reddy, and Levinson beam theories, are reformulated using strain or stress gradient elasticity theory and have been used to obtain natural frequencies of vibration [21]. Natural frequencies of both Euler-Bernoulli beam model (EBM) and TBM 
tend to decrease due to nonlocal effects [22]. However, Di Paola et al. [23] presented the dynamics of a TBM within a mechanically based approach to nonlocal elasticity. They showed that the natural frequencies tend to increase with the internal length and are larger than the corresponding classical theories. Xu et al. [24] found a special frequency $w_{\mathrm{o}}$ in a series of resonant frequencies from which some noncoaxial vibrations will occur. Xu et al. [25] employed strain gradient elasticity with internal inertia and found that, for higher vibrational modes, both frequencies and amplitude ratios deviate from the predictions of classical elasticity.

Concerning the numerical methods for obtaining free vibrations of CNT, Adali [26] presented a variational formulation for free and forced vibrations. Shakouri et al. [27] presented an approximate approach through the Galerkin method. Eltaher et al. [28] used a finite element method for the study of vibrations in Euler-Bernoulli nanobeams. Alotta et al. [29] presented a finite element method for nonlocal TBM that relies on the idea that nonlocal effects consist of long-range volume forces and moments exchanged by nonadjacent beam segments.

In this work, the higher-order beam model (HBM) developed by Ambrosini [30] and Ambrosini and de Borbón [11] is extended, including the nonlocal scale effect. A finite beam element is developed and the element stiffness and mass matrix are presented. The numerical examples allow studying the influence of the scale parameter for different aspect ratios and boundary conditions.

\section{Analysis and methodology}

\subsection{Nonlocal theory}

According to Eringen [15], the stress at a reference point $r$ not only depends on the strain tensor at the point $r$ but also on the strain tensor at all other points $r^{\prime}$ of the body. The nonlocal stress tensor can be expressed as

$$
\boldsymbol{\sigma}(r)=\int_{V} \lambda\left(\left|r-r^{\prime}\right|, \alpha\right) \boldsymbol{t}\left(r^{\prime}\right) d V\left(r^{\prime}\right), \forall r \in V
$$

where $t(r)$ is the macroscopic stress tensor and $\lambda\left(\left|r-r^{\prime}\right|, \alpha\right)$ is the nonlocal modulus or attenuation function that is a function of the distance in Euclidean form $\left|r-r^{\prime}\right|$ and a material constant $a$. $a$ is defined as $e_{0} a / l$, which depends on the internal characteristic length $a$ (lattice parameter, granular size, and distance between $\mathrm{C}-\mathrm{C}$ bonds) and the external characteristic length $l$ (crack length and wave length). The $e_{0}$ parameter is a dimensionless constant appropriate to each material, which can be estimated by tests or numerical simulations. There is no unique value of $e_{0}$ for CNTs because it is related to the complex internal microstructure and is supposed to depend on both the size and the chirality of CNT.

The kernel function $\lambda\left(\left|r-r^{\prime}\right|, \alpha\right)$ is given by [15] as

$$
\lambda\left(\left|r-r^{\prime}\right|, \alpha\right)=\left(2 \pi l^{2} \alpha^{2}\right)^{-1} K_{0}\left(\frac{\sqrt{r \cdot r}}{l \alpha}\right)
$$

where $K_{0}$ is the modified Bessel function. By combining Eqs. (1) and (2), the constitutive relations for elastic solids may be obtained as

$$
\begin{aligned}
& {\left[1-\left(e_{0} a\right)^{2} \nabla^{2}\right] \boldsymbol{\sigma}=\boldsymbol{t}} \\
& \boldsymbol{t}=\boldsymbol{C}: \boldsymbol{\varepsilon}
\end{aligned}
$$

where $\nabla^{2}$ is the Laplacian operator, $e$ is the strain tensor, and $C$ is the fourth-order elasticity tensor. When the nonlocal parameter $e_{0} a$ is equal to zero, the constitutive equations of the classical theory are recovered.

\subsection{Governing equations of motion}

The nonlocal effect is incorporated to the continuum model developed in Ref. [30]. This continuum model is a HBM for thin-walled beams, which takes into account shear deformations and rotatory inertia in the differential equations and internal forces. It can be used in open section beams and closed sections as well. Because the beams have a predominant dimension over the other two, the constitutive relation to the nonlocal effects is stated as

$$
\sigma(z)-\left(e_{0} a\right)^{2} \frac{\partial^{2} \sigma(z)}{\partial z^{2}}=E \varepsilon(z)
$$

where $E$ is the Young's modulus.

A left-handed rectangular global coordinates system $(x, y, z)$ shown in Figure 1 is adopted. The associated displacements are designated as $x, h$, and $z$.

The Euler-Lagrange equations of Ref. [30] can be expressed as

$$
\begin{aligned}
\frac{\partial M_{x}}{\partial z} & =\rho F\left(\frac{\partial^{2} \eta}{\partial t^{2}}+a_{x} \frac{\partial^{2} \theta}{\partial t^{2}}\right)-q_{y}-\rho J_{x}\left(\frac{\partial^{3} \eta}{\partial z \partial t^{2}}-\frac{\partial^{2} \gamma_{m y}}{\partial t^{2}}\right), \\
\frac{\partial M_{y}}{\partial z} & =\rho F\left(-\frac{\partial^{2} \xi}{\partial t^{2}}-a_{y} \frac{\partial^{2} \theta}{\partial t^{2}}\right)+q_{x}+\rho J_{y}\left(\frac{\partial^{3} \xi}{\partial z \partial t^{2}}-\frac{\partial^{2} \gamma_{m x}}{\partial t^{2}}\right), \\
\frac{\partial B}{\partial z} & =-\rho J_{\phi} \frac{\partial^{4} \theta}{\partial z \partial t^{2}}-G J_{d} \frac{\partial \theta}{\partial z}+\rho F a_{y} \frac{\partial^{2} \xi}{\partial t^{2}}-\rho F a_{x} \frac{\partial^{2} \eta}{\partial t^{2}}+\rho F r^{2} \frac{\partial^{2} \theta}{\partial t^{2}}+m_{a}
\end{aligned}
$$




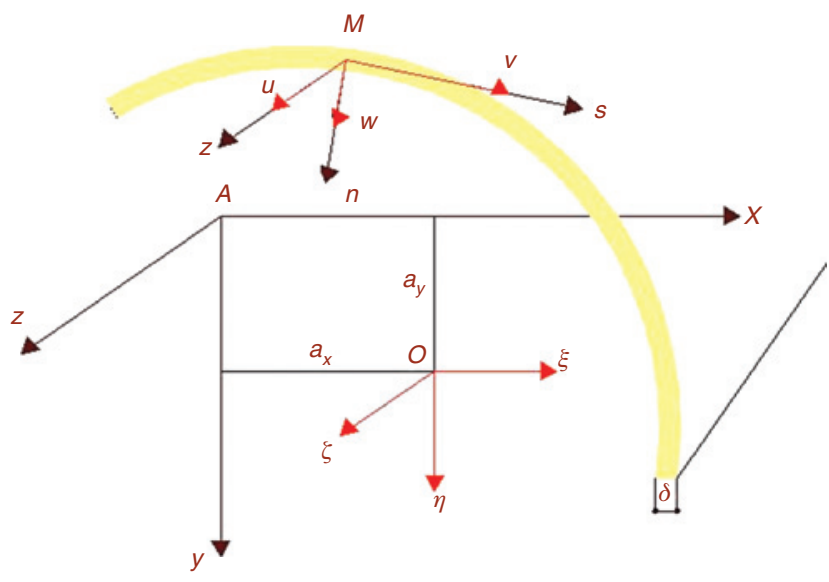

Figure 1: Coordinate systems and associated displacements.

where $M_{x}$ and $M_{y}$ are the flexural moments; $B$ is the bimoment; $r$ is the mass density; $F$ is the cross-section; $J_{x}$ and $J_{y}$ are the moments of inertia about the principal axes; $J_{d}$ is the torsional modulus; $J_{j}$ is the sectorial moment of inertia; $a_{x}$ and $a_{y}$ are the shear center coordinates; $q$ is the twist about $z$; and $q_{x}, q_{y}$, and $m_{a}$ are the external loads per unit length. $G_{m x}$ and $g_{m y}$ are given by

$$
\begin{aligned}
& \gamma_{m x}=\frac{Q_{x}}{k_{x} F G}, \\
& \gamma_{m y}=\frac{Q_{y}}{k_{y} F G}
\end{aligned}
$$

where $Q_{x}$ and $Q_{y}$ are the shear forces, $k_{x}$ and $k_{y}$ are the Cowper coefficients, and $G$ is the shear modulus. Finally,

$$
r^{2}=a_{x}^{2}+a_{y}^{2}+\frac{J_{x}+J_{y}}{F}
$$

The expressions for the flexural moments and bimoment are

$$
\begin{aligned}
& M_{x}-\left(e_{0} a\right)^{2} \frac{\partial^{2} M_{x}}{\partial z^{2}}=-E J_{x}\left(\frac{\partial^{2} \eta}{\partial z^{2}}-\frac{\partial \gamma_{m y}}{\partial z}\right), \\
& M_{y}-\left(e_{0} a\right)^{2} \frac{\partial^{2} M_{y}}{\partial z^{2}}=E J_{y}\left(\frac{\partial^{2} \xi}{\partial z^{2}}-\frac{\partial \gamma_{m x}}{\partial z}\right), \\
& B-\left(e_{0} a\right)^{2} \frac{\partial^{2} B}{\partial z^{2}}=-E J_{\varphi} \frac{\partial^{2} \theta}{\partial z^{2}},
\end{aligned}
$$

Considering that $\mu=\left(e_{0} a\right)^{2}$, deriving Eqs. (8) and combining Eqs. (8) and (5), the differential equations of motion are obtained as

$$
\begin{aligned}
& E J_{x}\left(\frac{\partial^{4} \eta}{\partial z^{4}}-\frac{\partial^{3} \gamma_{m y}}{\partial^{3} z}\right)+\rho F\left(\frac{\partial^{2} \eta}{\partial t^{2}}-a_{x} \frac{\partial^{2} \theta}{\partial t^{2}}\right)-\rho J_{x}\left(\frac{\partial^{4} \eta}{\partial z^{2} \partial t^{2}}-\frac{\partial^{3} \gamma_{m y}}{\partial z \partial t^{2}}\right) \\
& -\mu \rho F\left(\frac{\partial^{4} \eta}{\partial z^{2} \partial t^{2}}-a_{x} \frac{\partial^{2} \theta}{\partial z^{2} \partial t^{2}}\right)+\mu \rho J_{x}\left(\frac{\partial^{6} \eta}{\partial z^{4} \partial t^{2}}-\frac{\partial^{5} \gamma_{m y}}{\partial z^{3} \partial t^{2}}\right)=q_{y}-\mu \frac{\partial^{2} q_{y}}{\partial z^{2}} \\
& E J_{y}\left(\frac{\partial^{4} \xi}{\partial z^{4}}-\frac{\partial^{3} \gamma_{m x}}{\partial^{3} z}\right)+\rho F\left(\frac{\partial^{2} \xi}{\partial t^{2}}+a_{y} \frac{\partial^{2} \theta}{\partial t^{2}}\right)-\rho J_{y}\left(\frac{\partial^{4} \xi}{\partial z^{2} \partial t^{2}}-\frac{\partial^{3} \gamma_{m x}}{\partial z \partial t^{2}}\right) \\
& -\mu \rho F\left(\frac{\partial^{4} \xi}{\partial z^{2} \partial t^{2}}+a_{y} \frac{\partial^{4} \theta}{\partial z^{2} \partial t^{2}}\right)+\mu \rho J_{y}\left(\frac{\partial^{6} \xi}{\partial z^{4} \partial t^{2}}-\frac{\partial^{5} \gamma_{m x}}{\partial z^{3} \partial t^{2}}\right)=q_{x}-\mu \frac{\partial^{2} q_{x}}{\partial z^{2}}, \\
& E J_{\varphi} \frac{\partial^{4} \theta}{\partial z^{4}}-G J_{d} \frac{\partial^{2} \theta}{\partial z^{2}}-\rho J_{\varphi} \frac{\partial^{4} \theta}{\partial z^{2} \partial t^{2}}+\rho F a_{y} \frac{\partial^{2} \xi}{\partial t^{2}}-\rho F a_{x} \frac{\partial^{2} \eta}{\partial t^{2}}+\rho F r^{2} \frac{\partial^{2} \theta}{\partial t^{2}} \\
& +\mu \rho J_{\varphi} \frac{\partial^{6} \theta}{\partial z^{4} \partial t^{2}}+\mu G J_{d} \frac{\partial^{4} \theta}{\partial z^{4}}-\mu \rho F\left(a_{y} \frac{\partial^{4} \xi}{\partial z^{2} \partial t^{2}}+a_{x} \frac{\partial^{4} \eta}{\partial z^{2} \partial t^{2}}\right. \\
& \left.-r^{2} \frac{\partial^{4} \theta}{\partial z^{2} \partial t^{2}}\right)=m_{a}-\mu \frac{\partial^{2} m_{a}}{\partial z^{2}}
\end{aligned}
$$

\subsection{Nonlocal model for double-walled carbon nanotubes (DWCNTs)}

In double-walled carbon nanotubes (DWCNTs; or MWCNT), the deflections of the tubes in the $x$ and $y$ directions are coupled through the van der Waals (vdW) intertube interaction pressure $p$. The torsional deflections remain uncoupled. Because the inner and outer tubes are originally concentric and the vdW interaction is determined by the interlayer spacing between the tubes, the net vdW interaction pressure remains zero for each tube if they vibrate coaxially and share the same deflection curve. For a multiwalled beam model, the tubes are described by individual deflection curves that may not be coincident. Therefore, for small amplitude noncoaxial linear vibration, the vdW interaction pressure at any position $x$ (or $h$ ) between two tubes depends linearly on the difference of their deflection curves at that position [31]

$$
p=c_{1}\left(\xi_{2}-\xi_{1}\right)
$$

where $c$ is the vdW interaction coefficient between the tubes. The coefficient $c$ can be estimated as [32]:

$$
c_{k}=\frac{320\left(2 r_{k}\right)}{0.16 a^{2}} \mathrm{erg} / \mathrm{cm}^{2} \quad k=1,2, \ldots, n
$$

where $\alpha=0.142 \mathrm{~nm}$ and $r_{k}$ is the radius of the tube considered. Other expressions of $c_{k}$ are available in the literature, but, as proved in Ref. [33], the choice of $c_{k}$ widely influences the noncoaxial frequencies. 
For concentric DWCNTs, $A$ and $O$ (Figure 1) are coincident, but in some cases the nanotubes could have initial deformations due to, for example, defects, external actions, and attached masses in CNT-based resonators. In this case, the general model should be applied. When $A$ and $O$ are coincident, as a result of the axial symmetry of nanotubes, only displacement $x$ is considered in plane $x y$. Hence, considering Eqs. (9) to (11), the nonlocal differential equations of motion for DWCNT are expressed as

$$
\begin{aligned}
& E J_{y}\left(\frac{\partial^{4} \xi_{1}}{\partial z^{4}}-\frac{\partial^{3} \gamma_{1 m x}}{\partial^{3} z}\right)+\rho F\left(\frac{\partial^{2} \xi_{1}}{\partial t^{2}}+a_{y} \frac{\partial^{2} \theta_{1}}{\partial t^{2}}\right)-\rho J_{y}\left(\frac{\partial^{4} \xi_{1}}{\partial z^{2} \partial t^{2}}-\frac{\partial^{3} \gamma_{1 m x}}{\partial z \partial t^{2}}\right) \\
& -\mu \rho F\left(\frac{\partial^{4} \xi_{1}}{\partial z^{2} \partial t^{2}}+a_{y} \frac{\partial^{4} \theta_{1}}{\partial z^{2} \partial t^{2}}\right)+\mu \rho J_{y}\left(\frac{\partial^{6} \xi_{1}}{\partial z^{4} \partial t^{2}}-\frac{\partial^{5} \gamma_{1 m x}}{\partial z^{3} \partial t^{2}}\right) \\
& =c_{1}\left(\xi_{2}-\xi\right)-\mu c_{1}\left(\frac{\partial^{2} \xi_{2}}{\partial z^{2}}-\frac{\partial^{2} \xi_{1}}{\partial z^{2}}\right) \text {, } \\
& E J_{y}\left(\frac{\partial^{4} \xi_{2}}{\partial z^{4}}-\frac{\partial^{3} \gamma_{2 m x}}{\partial^{3} z}\right)+\rho F\left(\frac{\partial^{2} \xi_{2}}{\partial t^{2}}+a_{y} \frac{\partial^{2} \theta_{2}}{\partial t^{2}}\right)-\rho J_{y}\left(\frac{\partial^{4} \xi_{2}}{\partial z^{2} \partial t^{2}}-\frac{\partial^{3} \gamma_{2 m x}}{\partial z \partial t^{2}}\right) \\
& -\mu \rho F\left(\frac{\partial^{4} \xi_{2}}{\partial z^{2} \partial t^{2}}+a_{y} \frac{\partial^{4} \theta_{2}}{\partial z^{2} \partial t^{2}}\right)+\mu \rho J_{y}\left(\frac{\partial^{6} \xi_{2}}{\partial z^{4} \partial t^{2}}-\frac{\partial^{5} \gamma_{2 m x}}{\partial z^{3} \partial t^{2}}\right) \\
& =-c_{1}\left(\xi_{2}-\xi_{1}\right)+\mu c_{1}\left(\frac{\partial^{2} \xi_{2}}{\partial z^{2}}-\frac{\partial^{2} \xi_{1}}{\partial z^{2}}\right) \text {, } \\
& E J_{\varphi} \frac{\partial^{4} \theta_{1}}{\partial z^{4}}-G J_{d} \frac{\partial^{2} \theta_{1}}{\partial z^{2}}-\rho J_{\varphi} \frac{\partial^{4} \theta_{1}}{\partial z^{2} \partial t^{2}}+\rho F a_{y} \frac{\partial^{2} \xi_{1}}{\partial t^{2}}-\rho F a_{x} \frac{\partial^{2} \eta_{1}}{\partial t^{2}} \\
& +\rho F r^{2} \frac{\partial^{2} \theta_{1}}{\partial t^{2}}+\mu \rho J_{\varphi} \frac{\partial^{6} \theta_{1}}{\partial z^{4} \partial t^{2}}+\mu G J_{d} \frac{\partial^{4} \theta_{1}}{\partial z^{4}} \\
& -\mu \rho F\left(a_{y} \frac{\partial^{4} \xi_{1}}{\partial z^{2} \partial t^{2}}+a_{x} \frac{\partial^{4} \eta_{1}}{\partial z^{2} \partial t^{2}}-r^{2} \frac{\partial^{4} \theta_{1}}{\partial z^{2} \partial t^{2}}\right)=0 \\
& E J_{\varphi} \frac{\partial^{4} \theta_{2}}{\partial z^{4}}-G J_{d} \frac{\partial^{2} \theta_{2}}{\partial z^{2}}-\rho J_{\varphi} \frac{\partial^{4} \theta_{2}}{\partial z^{2} \partial t^{2}}+\rho F a_{y} \frac{\partial^{2} \xi_{2}}{\partial t^{2}}-\rho F a_{x} \frac{\partial^{2} \eta_{2}}{\partial t^{2}} \\
& +\rho F r^{2} \frac{\partial^{2} \theta_{2}}{\partial t^{2}}+\mu \rho J_{\varphi} \frac{\partial^{6} \theta_{2}}{\partial z^{4} \partial t^{2}}+\mu G J_{d} \frac{\partial^{4} \theta_{2}}{\partial z^{4}} \\
& -\mu \rho F\left(a_{y} \frac{\partial^{4} \xi_{2}}{\partial z^{2} \partial t^{2}}+a_{x} \frac{\partial^{4} \eta_{2}}{\partial z^{2} \partial t^{2}}-r^{2} \frac{\partial^{4} \theta_{2}}{\partial z^{2} \partial t^{2}}\right)=0
\end{aligned}
$$

\subsection{Nonlocal model for DWCNT}

A finite beam element is developed from the Galerkin method for differential operators. The differentials equations must be replaced by a weak form, which is equivalent $[34,35]$. Following the methodology presented by de Borbón et al. [36], the mass and stiffness matrix for nonlocal formulation are obtained.

\subsubsection{Variational formulation and beam element}

First, the equations of motion are expressed in terms of displacements eliminating the shear strains:

$$
\begin{aligned}
& E J_{y 1} \frac{\partial^{4} \xi_{1}}{\partial z^{4}}+\rho F_{1}\left(\frac{\partial^{2} \xi_{1}}{\partial t^{2}}+a_{y} \frac{\partial^{2} \theta_{1}}{\partial t^{2}}\right)-\frac{E J_{y 1} \rho}{k_{x} G}\left(\frac{\partial^{4} \xi_{1}}{\partial z^{2} \partial t^{2}}+a_{y} \frac{\partial^{4} \theta_{1}}{\partial z^{2} \partial t^{2}}\right) \\
& -\rho J_{y 1} \frac{\partial^{4} \xi_{1}}{\partial z^{2} \partial t^{2}}+\frac{\rho^{2} J_{1 y}}{k_{x} G}\left(\frac{\partial^{4} \xi_{1}}{\partial t^{4}}+a_{y} \frac{\partial^{4} \theta_{1}}{\partial t^{4}}\right)-\mu \rho F_{1}\left(\frac{\partial^{4} \xi_{1}}{\partial z^{2} \partial t^{2}}+a_{y} \frac{\partial^{4} \theta_{1}}{\partial z^{2} \partial t^{2}}\right) \\
& +\mu \rho J_{y} \frac{\partial^{6} \xi_{1}}{\partial z^{4} \partial t^{2}}-\frac{\mu \rho^{2} J_{1 y}}{k_{x} G}\left(\frac{\partial^{6} \xi_{1}}{\partial z^{2} \partial t^{4}}+a_{y} \frac{\partial^{6} \theta_{1}}{\partial z^{2} \partial t^{4}}\right) \\
& -c_{1}\left(\xi_{2}-\xi_{1}\right)+\mu c_{1}\left(\frac{\partial^{2} \xi_{2}}{\partial z^{2}}-\frac{\partial^{2} \xi_{1}}{\partial z^{2}}\right)=0, \\
& E J_{y 2} \frac{\partial^{4} \xi_{2}}{\partial z^{4}}+\rho F_{2}\left(\frac{\partial^{2} \xi_{2}}{\partial t^{2}}+a_{y} \frac{\partial^{2} \theta_{2}}{\partial t^{2}}\right)-\frac{E J_{y 2} \rho}{k_{x} G}\left(\frac{\partial^{4} \xi_{2}}{\partial z^{2} \partial t^{2}}+a_{y} \frac{\partial^{4} \theta_{2}}{\partial z^{2} \partial t^{2}}\right) \\
& -\rho J_{y 2} \frac{\partial^{4} \xi_{2}}{\partial z^{2} \partial t^{2}}+\frac{\rho^{2} J_{2 y}}{k_{x} G}\left(\frac{\partial^{4} \xi_{2}}{\partial t^{4}}+a_{y} \frac{\partial^{4} \theta_{2}}{\partial t^{4}}\right)-\mu \rho F_{2}\left(\frac{\partial^{4} \xi_{2}}{\partial z^{2} \partial t^{2}}+a_{y} \frac{\partial^{4} \theta_{2}}{\partial z^{2} \partial t^{2}}\right) \\
& +\mu \rho J_{2 y} \frac{\partial^{6} \xi_{2}}{\partial z^{4} \partial t^{2}}-\frac{\mu \rho^{2} J_{2 y}}{k_{x} G}\left(\frac{\partial^{6} \xi_{2}}{\partial z^{2} \partial t^{4}}+a_{y} \frac{\partial^{6} \theta_{2}}{\partial z^{2} \partial t^{4}}\right) \\
& +c_{1}\left(\xi_{2}-\xi_{1}\right)-\mu c_{1}\left(\frac{\partial^{2} \xi_{2}}{\partial z^{2}}-\frac{\partial^{2} \xi_{1}}{\partial z^{2}}\right)=0,
\end{aligned}
$$

$E J_{\varphi} \frac{\partial^{4} \theta_{1}}{\partial z^{4}}-G J_{d} \frac{\partial^{2} \theta_{1}}{\partial z^{2}}-\rho J_{\varphi} \frac{\partial^{4} \theta_{1}}{\partial z^{2} \partial t^{2}}+\rho F a_{y} \frac{\partial^{2} \xi_{1}}{\partial t^{2}}-\rho F a_{x} \frac{\partial^{2} \eta_{1}}{\partial t^{2}}$

$+\rho F r^{2} \frac{\partial^{2} \theta_{1}}{\partial t^{2}}+\mu \rho J_{\varphi} \frac{\partial^{6} \theta_{1}}{\partial z^{4} \partial t^{2}}+\mu G J_{d} \frac{\partial^{4} \theta_{1}}{\partial z^{4}}$

$-\mu \rho F\left(a_{y} \frac{\partial^{4} \xi_{1}}{\partial z^{2} \partial t^{2}}+a_{x} \frac{\partial^{4} \eta_{1}}{\partial z^{2} \partial t^{2}}-r^{2} \frac{\partial^{4} \theta_{1}}{\partial z^{2} \partial t^{2}}\right)=0$

$E J_{\varphi} \frac{\partial^{4} \theta_{2}}{\partial z^{4}}-G J_{d} \frac{\partial^{2} \theta_{2}}{\partial z^{2}}-\rho J_{\varphi} \frac{\partial^{4} \theta_{2}}{\partial z^{2} \partial t^{2}}+\rho F a_{y} \frac{\partial^{2} \xi_{2}}{\partial t^{2}}-\rho F a_{x} \frac{\partial^{2} \eta_{2}}{\partial t^{2}}$

$+\rho F r^{2} \frac{\partial^{2} \theta_{2}}{\partial t^{2}}+\mu \rho J_{\varphi} \frac{\partial^{6} \theta_{2}}{\partial z^{4} \partial t^{2}}+\mu G J_{d} \frac{\partial^{4} \theta_{2}}{\partial z^{4}}$

$-\mu \rho F\left(a_{y} \frac{\partial^{4} \xi_{2}}{\partial z^{2} \partial t^{2}}+a_{x} \frac{\partial^{4} \eta_{2}}{\partial z^{2} \partial t^{2}}-r^{2} \frac{\partial^{4} \theta_{2}}{\partial z^{2} \partial t^{2}}\right)=0$

Equations (14) and (15) along with the boundary conditions represent the strong form of the problem. From the Galerkin method for differential operators,

$$
\int_{0}^{l} v_{i} A\left(u_{i}\right) d z=0 \quad i=1, \ldots, 3
$$

where $A\left(u_{i}\right)$ represents the differential equations with $u_{1}=x, u_{2}=h$, and $u_{3}=q$. The weak problem is to find: 


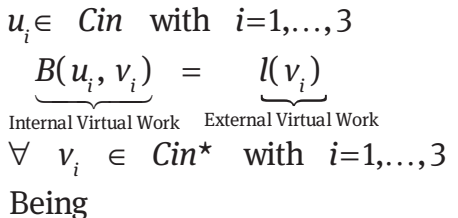

$$
\begin{gathered}
\operatorname{Cin}=\left\{\begin{array}{l}
u_{i}(z, t) \in L_{2}(\Omega), \frac{\partial u_{i}(z, t)}{\partial z} \in L_{2}(\Omega) \\
\text { and } \frac{\partial^{2} u_{i}(z, t)}{\partial z^{2}} \in L_{2}(\Omega)
\end{array}\right\} \\
\operatorname{Cin}^{\star}=\left\{\begin{array}{l}
v_{i}(z, t) \in L_{2}(\Omega), \frac{\partial v_{i}(z, t)}{\partial z} \in L_{2}(\Omega) \\
\text { and } \frac{\partial^{2} v_{i}(z, t)}{\partial z^{2}} \in L_{2}(\Omega)
\end{array}\right\}
\end{gathered}
$$

where the bilinear operator $B\left(u_{i}, v_{i}\right)$ has mass and stiffness terms and the lineal operator $l\left(v_{i}\right)$ has the boundary conditions. The chosen trial functions $u_{i}$ have the following characteristics:

$$
\begin{gathered}
u_{1}=\xi(z, t)=\alpha_{1}(t) \xi(z) \\
u_{2}=\eta(z, t)=\alpha_{2}(t) \eta(z) \\
u_{3}=\theta(z, t)=\alpha_{3}(t) \theta(z) \\
\alpha_{i}=e^{i \omega t} \quad i=1, \ldots, 3
\end{gathered}
$$

The Hermite cubic polynomials are chosen as shape functions to interpolate the displacements of $u_{i}$ and $v_{i}$ (Figure 2).

The displacements of an element of length $h_{e}$ are defined as

$$
u_{i}=v_{i}=\boldsymbol{N}_{i}^{e} a_{i}^{e}
$$

where $N_{i}^{e}$ is the shape functions matrix for an element and $a_{i}^{e}$ is the nodal vector with the degree of freedom of the element. Using the following nomenclature to indicate first and second derivate of the shape functions,

$$
\begin{aligned}
& G^{e}=\left(\frac{\partial N_{1}^{e}(s)}{\partial s} \frac{\partial N_{2}^{e}(s)}{\partial s} \frac{\partial N_{3}^{e}(s)}{\partial s} \frac{\partial N_{4}^{e}(s)}{\partial s}\right)\left(\frac{d s}{d z}\right) \\
& B^{e}=\left(\frac{\partial^{2} N_{1}^{e}(s)}{\partial s^{2}} \frac{\partial^{2} N_{2}^{e}(s)}{\partial s^{2}} \frac{\partial^{2} N_{3}^{e}(s)}{\partial s^{2}} \frac{\partial^{2} N_{4}^{e}(s)}{\partial s^{2}}\right)\left(\frac{d s}{d z}\right)^{2}
\end{aligned}
$$

The expressions of the stiffness and mass element matrices are finally presented with the nonlocal formulation. The mass matrix is shown like a sum of matrices only with the purpose of clarifying its content. The stiffness and mass element matrices have been ordered showing the flexural-torsional coupled terms.

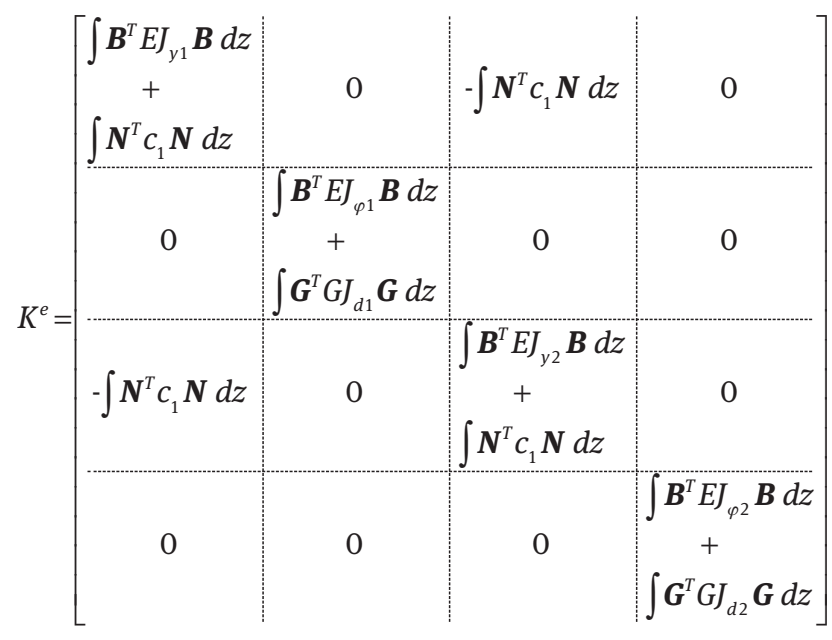

The first two matrixes $M_{1}^{e}$ and $M_{n}{ }_{1}^{e}$ have the terms of mass of classical and nonlocal theories, respectively. The $M_{2}^{e}$ matrix has the terms that affect the accelerations originated by the shear deformations. Finally, $M_{3}{ }^{e}$ and $\mathrm{Mnl}_{3}{ }^{e}$ have terms that affect the accelerations originated by the shear deformations but associated to fourth-order time derivative. In slender beams, this last matrix can be neglected [37].
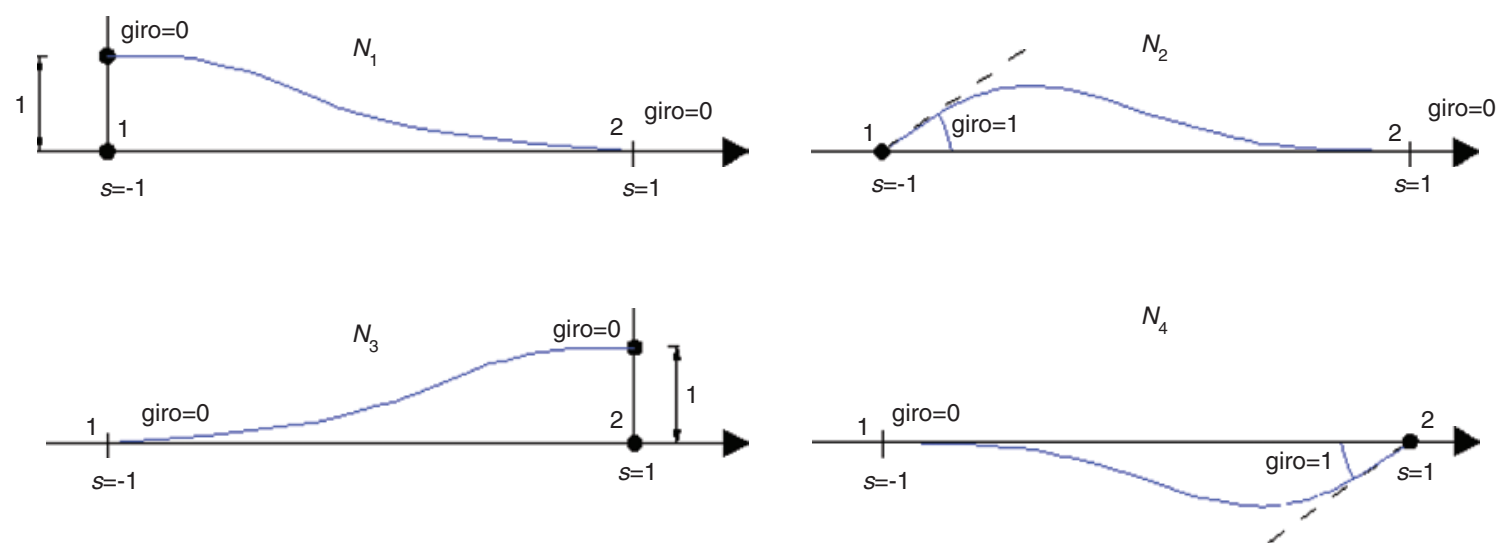

Figure 2: Shape functions: Hermite cubic polynomials. 


\begin{tabular}{|c|c|c|c|}
\hline $\begin{array}{c}\int \boldsymbol{N}^{T} m_{1} \boldsymbol{N} d z \\
+ \\
\int \boldsymbol{G}^{T} \rho J_{y 1} \boldsymbol{G} d z\end{array}$ & $\int \boldsymbol{N}^{T} m_{1} a_{y 1} \boldsymbol{N} d z$ & 0 & 0 \\
\hline $\int \boldsymbol{N}^{T} m_{1} a_{y 1} \boldsymbol{N} d z$ & $\begin{array}{c}\int \boldsymbol{N}^{T} m_{1} r_{1}^{2} \boldsymbol{N} d z \\
+ \\
\int \boldsymbol{G}^{T} \rho J_{\varphi 1} \boldsymbol{G} d z\end{array}$ & 0 & 0 \\
\hline 0 & 0 & $\begin{array}{c}\int \boldsymbol{N}^{T} m_{2} \boldsymbol{N} d z \\
+ \\
\int \boldsymbol{G}^{T} \rho J_{y 2} \boldsymbol{G} d z\end{array}$ & $\int \boldsymbol{N}^{T} m_{2} a_{y 2} \boldsymbol{N} d z$ \\
\hline 0 & 0 & $\int \boldsymbol{N}^{T} m_{2} a_{y 2} \boldsymbol{N} d z$ & $\begin{array}{c}\int \boldsymbol{N}^{T} m_{2} r_{2}^{2} \boldsymbol{N} d z \\
+ \\
\int \boldsymbol{G}^{T} \rho J_{\varphi 2} \boldsymbol{G} d z\end{array}$ \\
\hline
\end{tabular}

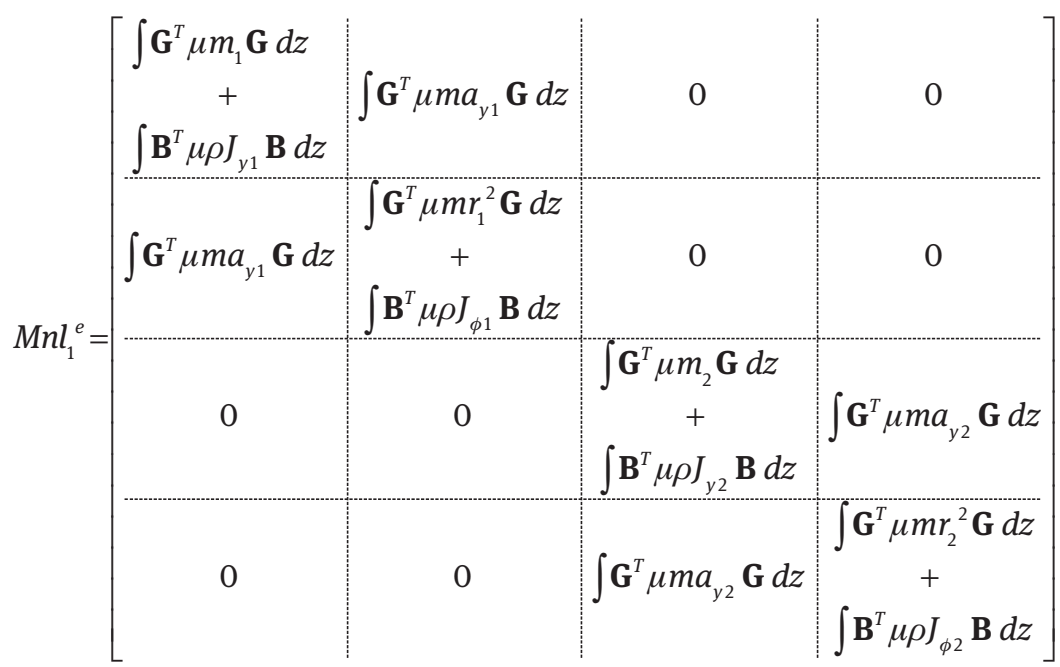

\begin{tabular}{|c|c|c|c|}
\hline $\int \boldsymbol{G}^{T} \frac{m_{1} E J_{y 1}}{k_{x} F_{1} G} \boldsymbol{G} d z$ & $\int \boldsymbol{G}^{T} \frac{m_{1} E J_{y 1}}{k_{x} F_{1} G} a_{y 1} \boldsymbol{G} d z$ & 0 & 0 \\
\hline 0 & 0 & 0 & 0 \\
\hline 0 & 0 & $\int \boldsymbol{G}^{T} \frac{m_{2} E J_{y 2}}{k_{x} F_{2} G} \boldsymbol{G} d z$ & $\int \boldsymbol{G}^{T} \frac{m_{2} E J_{y 2}}{k_{x} F_{2} G} a_{y 2} \boldsymbol{G} d z$ \\
\hline 0 & 0 & 0 & 0 \\
\hline
\end{tabular}




$M_{3}^{e}=\left[\begin{array}{c|c|c|c}\int \boldsymbol{N}^{T} \frac{\rho J_{y 1} m_{1}}{k_{x} F_{1} G} \boldsymbol{N} d z & \int \boldsymbol{N}^{T} \frac{\rho J_{y 1} m_{1}}{k_{x} F_{1} G} a_{y 1} \boldsymbol{N} d z & 0 & 0 \\ \hdashline 0 & 0 & 0 & 0 \\ \hdashline 0 & 0 & \int \boldsymbol{N}^{T} \frac{\rho J_{y 2} m_{2}}{k_{x} F_{2} G} \boldsymbol{N} d z & \int \boldsymbol{N}^{T} \frac{\rho J_{y 2} m_{2}}{k_{x} F_{2} G} a_{y 2} \boldsymbol{N} d z \\ \hdashline 0 & 0 & 0\end{array}\right]$

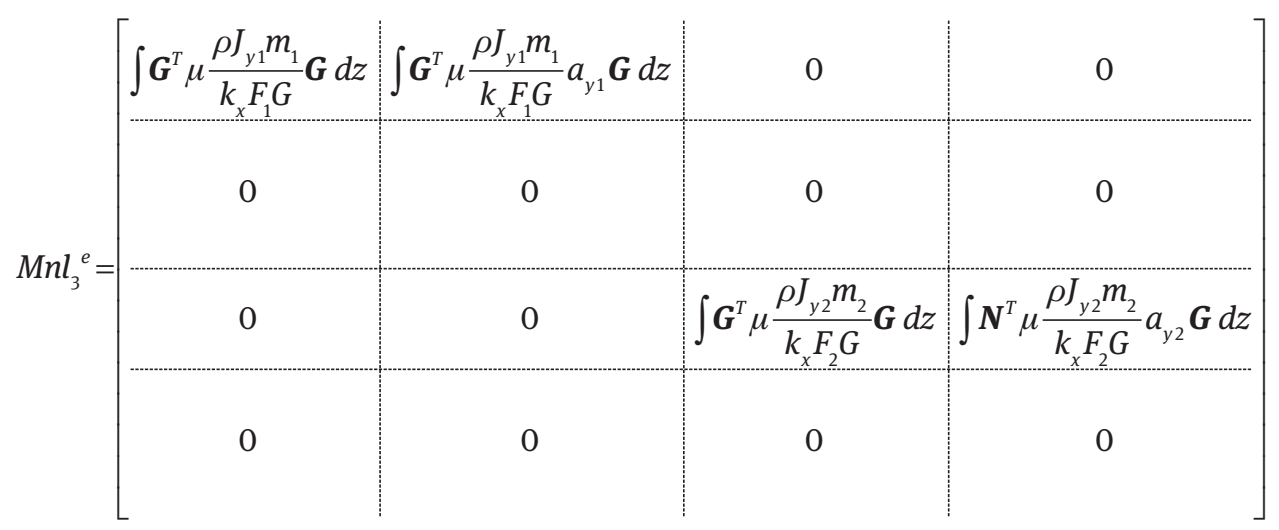

\subsubsection{Eigenvalue problem}

The eigenvalue problem is

$$
\left(K-\lambda M_{1}-\lambda M_{2}-\lambda M n l_{2}+\lambda^{2} M_{3}+\lambda^{2} M n l_{3}\right) a=0
$$

where

$$
\lambda=\omega^{2}
$$

If it is called

$$
b=\lambda a
$$

Equation (31) can be expressed as

$$
K a-\lambda a M_{1}-\lambda a M_{2}-\lambda a M n l_{2}+\lambda b M_{3}+\lambda b M n l_{3}=0
$$

Equation (31) can be expressed in matrix form as

$$
\left[\begin{array}{ll}
K & 0 \\
0 & I
\end{array}\right]\left[\begin{array}{l}
a \\
b
\end{array}\right]-\lambda\left[\begin{array}{cc}
M_{1}+M_{2}+M n l_{2} & -M_{3}-M n l_{3} \\
I & 0
\end{array}\right]\left[\begin{array}{l}
a \\
b
\end{array}\right]=0
$$

The angular frequency and the frequency can be found as

$$
\begin{gathered}
\omega=\sqrt{\lambda}, \\
f=\frac{\omega}{2 \pi}
\end{gathered}
$$

\section{Results and discussion}

(30) To validate the beam model and the methodology proposed, numerical examples are reproduced from Ehteshami and Hajabasi [38]. The model proposed was implemented in a finite element program considering that the mass and stiffness element matrices can be programmed and assembled in any computational frame (e.g. MATLAB). Afterward, the coaxial and noncoaxial frequencies are obtained for nanotubes of different aspect ratios. The influence of the nonlocal parameter, shear deformation, and boundary conditions is studied. 


\subsection{Model validation}

The nanotubes in consideration are simply supported and clamped. They have the following mechanical and geometric properties:

$$
\begin{aligned}
E & =1 \mathrm{TPa} ; r=2.3 \mathrm{~g} / \mathrm{cm}^{3} \\
t & =0.35 \mathrm{~nm} ; n=0.25 \\
d_{1} & =0.7 \mathrm{~nm} ; d_{2}=1.4 \mathrm{~nm} \\
\alpha & =e_{0} a / 1=0.1 ; 1 / d_{2}=10
\end{aligned}
$$

Ten beam elements are used. The obtained results for the natural frequency are presented in Tables 1 and 2.

For both DWCNTs, the results obtained with the proposed model are in excellent agreement with those of the referenced paper [38].

\subsection{Coaxial and noncoaxial frequencies}

To investigate the vibration characteristics of different aspect ratio nanotubes (10 and 20), numerical examples are carried out considering in the beam model the shear
Table 1: Natural frequency (THz). Nanotube simply supported.

\begin{tabular}{lrrr}
\hline & This paper & Ref. [38] & Difference (\%) \\
\hline$\alpha=0$ & 0.0745 & 0.0745 & 0.00 \\
$\alpha=0.1$ & 0.0714 & 0.0711 & 0.42 \\
\hline
\end{tabular}

Table 2: Natural frequency (THz). Nanotube clamped.

\begin{tabular}{lrrr}
\hline & This paper & Ref. [38] & Difference (\%) \\
\hline$\alpha=0$ & 0.169 & 0.169 & 0.00 \\
$\alpha=0.1$ & 0.160 & 0.159 & 0.63 \\
\hline
\end{tabular}

deformations (TBM) or not (EBM). The support conditions considered are simply supported, cantilever, clamped, and clamped-hinged. The nanotube has the same properties than the previous example. The results are presented in Tables 3-10. The nanotubes in consideration are simply supported and clamped.

\begin{tabular}{|c|c|c|c|c|c|c|c|c|}
\hline & \multicolumn{2}{|c|}{$1^{\circ}$ Coaxial } & \multicolumn{2}{|c|}{$2^{\circ}$ Coaxial } & \multicolumn{2}{|c|}{$3^{\circ}$ Coaxial } & \multicolumn{2}{|c|}{$1^{\circ}$ Noncoaxial } \\
\hline & EBM & TBM & EBM & TBM & EBM & TBM & EBM & TBM \\
\hline$\alpha=0$ & 0.0745 & 0.0730 & 0.297 & 0.275 & 0.650 & 0.564 & 1.222 & 1.205 \\
\hline$\alpha=0.1$ & 0.0714 & 0.0700 & 0.255 & 0.238 & 0.491 & 0.429 & 1.227 & 1.210 \\
\hline$\alpha=0.2$ & 0.0639 & 0.0628 & 0.191 & 0.179 & 0.324 & 0.285 & 1.237 & 1.226 \\
\hline$\alpha=0.3$ & 0.0554 & 0.0545 & 0.145 & 0.136 & 0.233 & 0.206 & 1.248 & 1.231 \\
\hline
\end{tabular}

The frequencies obtained considering the shear deformations (TBM) are lower than those obtained with

Table 3: Natural frequency (THz). Nanotube simply supported. $L=14 \mathrm{~nm}$.

\begin{tabular}{|c|c|c|c|c|c|c|c|c|}
\hline & \multicolumn{2}{|c|}{$1^{\circ}$ Coaxial } & \multicolumn{2}{|c|}{$2^{\circ}$ Coaxial } & \multicolumn{2}{|c|}{$3^{\circ}$ Coaxial } & \multicolumn{2}{|c|}{$1^{\circ}$ Noncoaxial } \\
\hline & EBM & TBM & EBM & TBM & EBM & TBM & EBM & TBM \\
\hline$\alpha=0$ & 0.0266 & 0.0263 & 0.166 & 0.156 & 0.459 & 0.402 & 1.221 & 1.215 \\
\hline$\alpha=0.1$ & 0.0260 & 0.0258 & 0.146 & 0.138 & 0.355 & 0.319 & 1.223 & 1.218 \\
\hline$\alpha=0.2$ & 0.0245 & 0.0243 & 0.113 & 0.108 & 0.244 & 0.224 & 1.227 & 1.223 \\
\hline$\alpha=0.3$ & 0.0225 & 0.0224 & 0.088 & 0.086 & 0.181 & 0.167 & 1.232 & 1.222 \\
\hline
\end{tabular}

Table 4: Natural frequency (THz). Nanotube simply supported. $L=28 \mathrm{~nm}$.

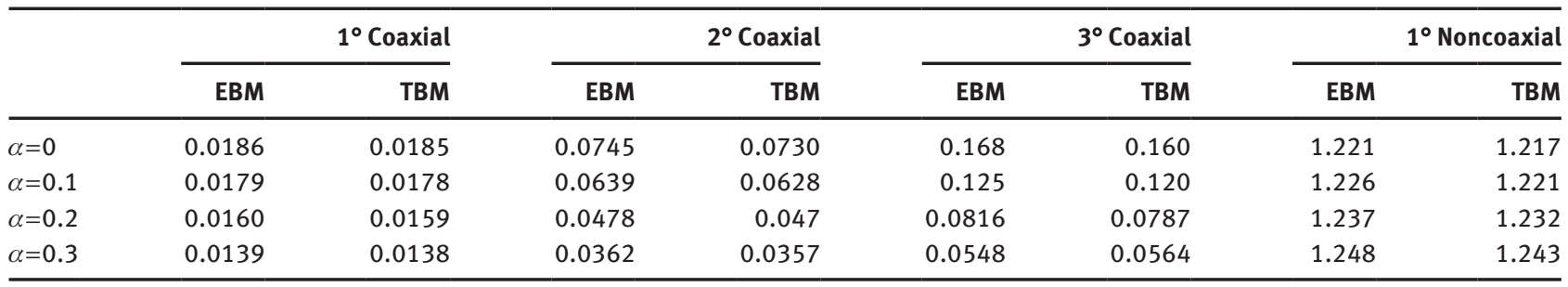

Table 5: Natural frequency (THz). Nanotube cantilever. $L=14 \mathrm{~nm}$. 
Table 6: Natural frequency (THz). Nanotube cantilever. $L=28 \mathrm{~nm}$.

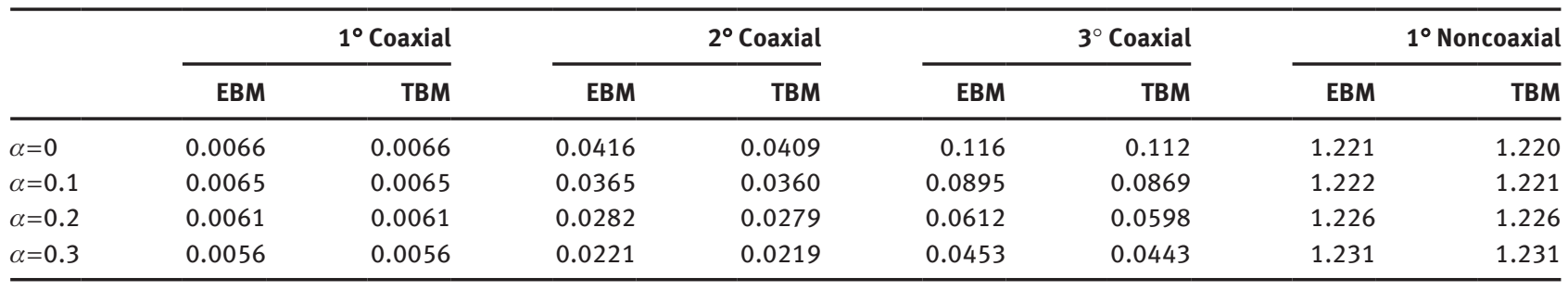

Table 7: Natural frequency (THz). Nanotube clamped. $L=14 \mathrm{~nm}$.

\begin{tabular}{|c|c|c|c|c|c|c|c|c|}
\hline & \multicolumn{2}{|c|}{$1^{\circ}$ Coaxial } & \multicolumn{2}{|c|}{$2^{\circ}$ Coaxial } & \multicolumn{2}{|c|}{$3^{\circ}$ Coaxial } & \multicolumn{2}{|c|}{$1^{\circ}$ Noncoaxial } \\
\hline & EBM & TBM & EBM & TBM & EBM & TBM & EBM & TBM \\
\hline$\alpha=0$ & 0.169 & 0.165 & 0.459 & 0.422 & 0.858 & 0.745 & 1.229 & 1.204 \\
\hline$\alpha=0.1$ & 0.160 & 0.156 & 0.387 & 0.353 & 0.644 & 0.541 & 1.234 & 1.208 \\
\hline$\alpha=0.2$ & 0.140 & 0.135 & 0.282 & 0.255 & 0.423 & 0.349 & 1.245 & 1.209 \\
\hline$\alpha=0.3$ & 0.119 & 0.114 & 0.211 & 0.190 & 0.305 & 0.250 & 1.254 & 1.210 \\
\hline
\end{tabular}

Table 8: Natural frequency (THz). Nanotube clamped. $L=28 \mathrm{~nm}$.

\begin{tabular}{|c|c|c|c|c|c|c|c|c|}
\hline & \multicolumn{2}{|c|}{$1^{\circ}$ Coaxial } & \multicolumn{2}{|c|}{$2^{\circ}$ Coaxial } & \multicolumn{2}{|c|}{$3^{\circ}$ Coaxial } & \multicolumn{2}{|c|}{$1^{\circ}$ Noncoaxial } \\
\hline & EBM & TBM & EBM & TBM & EBM & TBM & EBM & TBM \\
\hline$\alpha=0$ & 0.0423 & 0.0420 & 0.116 & 0.114 & 0.228 & 0.217 & 1.221 & 1.217 \\
\hline$\alpha=0.1$ & 0.0401 & 0.0398 & 0.0977 & 0.0952 & 0.166 & 0.157 & 1.227 & 1.221 \\
\hline$\alpha=0.2$ & 0.0351 & 0.0348 & 0.0709 & 0.0690 & 0.1 .07 & 0.101 & 1.238 & 1.232 \\
\hline$\alpha=0.3$ & 0.0297 & 0.0294 & 0.0530 & 0.0515 & 0.0765 & 0.0724 & 1.249 & 1.241 \\
\hline
\end{tabular}

Table 9: Natural frequency (THz). Nanotube clamped-hinged. $L=14 \mathrm{~nm}$.

\begin{tabular}{|c|c|c|c|c|c|c|c|c|}
\hline & \multicolumn{2}{|c|}{$1^{\circ}$ Coaxial } & \multicolumn{2}{|c|}{$2^{\circ}$ Coaxial } & \multicolumn{2}{|c|}{$3^{\circ}$ Coaxial } & \multicolumn{2}{|c|}{$1^{\circ}$ Noncoaxial } \\
\hline & EBM & TBM & EBM & TBM & EBM & TBM & EBM & TBM \\
\hline$\alpha=0$ & 0.116 & 0.114 & 0.374 & 0.345 & 0.753 & 0.652 & 1.225 & 1.204 \\
\hline$\alpha=0.1$ & 0.111 & 0.1 .08 & 0.318 & 0.294 & 0.566 & 0.485 & 1.230 & 1.209 \\
\hline$\alpha=0.2$ & 0.097 & 0.095 & 0.235 & 0.217 & 0.372 & 0.318 & 1.241 & 1.217 \\
\hline$\alpha=0.3$ & 0.083 & 0.081 & 0.178 & 0.164 & 0.268 & 0.229 & 1.251 & 1.225 \\
\hline
\end{tabular}

Table 10: Natural frequency (THz). Nanotube clamped-hinged $L=28 \mathrm{~nm}$.

\begin{tabular}{|c|c|c|c|c|c|c|c|c|}
\hline & \multicolumn{2}{|c|}{$1^{\circ}$ Coaxial } & \multicolumn{2}{|c|}{$2^{\circ}$ Coaxial } & \multicolumn{2}{|c|}{$3^{\circ}$ Coaxial } & \multicolumn{2}{|c|}{$1^{\circ}$ Noncoaxia } \\
\hline & EBM & TBM & EBM & TBM & EBM & TBM & EBM & TBM \\
\hline$\alpha=0$ & 0.0290 & 0.0289 & 0.0940 & 0.0923 & 0.197 & 0.188 & 1.221 & 1.218 \\
\hline$\alpha=0.1$ & 0.0277 & 0.0275 & 0.0800 & 0.0783 & 0.145 & 0.138 & 1.226 & 1.219 \\
\hline$\alpha=0.2$ & 0.0244 & 0.0243 & 0.0590 & 0.0577 & 0.0939 & 0.0899 & 1.237 & 1.232 \\
\hline$\alpha=0.3$ & 0.0208 & 0.0207 & 0.0445 & 0.0436 & 0.0671 & 0.0643 & 1.248 & 1.240 \\
\hline
\end{tabular}


EBM for all nonlocal parameters. This difference on the frequency value is more pronounced on short beams and higher modes. For a $14 \mathrm{~nm}$ length nanotube, the frequency decreases by $2 \%$ on the first mode and $13 \%$ on the third mode for all boundary conditions. However, for a $28 \mathrm{~nm}$ length nanotube, the frequency of the third mode decreases by approximately $4 \%$. For both lengths, when the nonlocal parameter increases, the influence of shear deformation decreases in simply supported and cantilever beams, increases in clamped beams, and remains constant in clamped-hinged beams. This behavior is better observed in short beams.

Concerning the nonlocal parameter, when this parameter increases, the natural frequencies of vibration decrease for both TBM and EBM. When $\alpha=0.3$, for the first mode, the frequency decreases by $25 \%$ in the simply supported beam, $15 \%$ in the cantilever beam, $30 \%$ in the clamped beam and $28 \%$ in the clamped-hinged beam. These percentages of reduction in the frequencies are similar for nanotubes of both aspect ratios. If the third mode is observed, the natural frequencies decrease by approximately $60 \%$ for all boundary conditions. Hence, the influence of the nonlocal parameter is crucial on higher modes. Even when $\alpha=0.1$, the frequencies of the first and third modes decrease by approximately $5 \%$ and $25 \%$, respectively.

Regarding the noncoaxial frequencies, as established in Ref. [11], it can be assumed that the noncoaxial frequencies are insensitive to shear deformations and independent of the boundary condition of the nanotube. When the nonlocal parameter increases, the noncoaxial frequencies also increase. For $\alpha=0.3$, the value rises by $2 \%$. However, a difference of $2 \%$ is negligible compared to a difference of $25 \%$ in the first mode.

The value of $e_{0}$ found in the literature differs with the nature of the problem and the model used for its calibration as can be seen in Table 11.

Zhang et al. [40] showed that the difference between the MD simulations and a nonlocal TBM was approximately 14\% for clamped-free beam and 12\% for clampedclamped beam considering a fixed nonlocal parameter. The values of Ansari and Sahmani [20] in Table 11 correspond to the Timoshenko nonlocal beam model. The values are above 1.31 for EBM and above 0.54 for the Reddy beam model. Arash and Ansari [41] compared a nonlocal shell model with MD simulations from Ref. [40] and obtained a completely different parameter. The results of the calibration of the parameter $e_{0}$ for the model proposed in this work for single-walled (SWCNT) and DWCNT is presented in Table 11 and Figures 3 and 4.

Both figures show an excellent agreement between the proposed beam model and the results from MD simulations. For DWCNT, better accuracy is obtained when the aspect ratio is $l / d>10$. The nonlocal parameter value increases as the aspect ratio increases and it depends also on the boundary conditions. The values of $\alpha=e_{0} a / l$ are $0.25-0.26$ for C-F SWCNT, 0.11-0.17 for C-C SWCNT, 0-0.22 for S-S DWCNT, 0-0.20 C-C DWCNT, and 0-0.21 C-S DWCNT. Eventually, it could be chosen a fix value of $a$, admitting an error.

Table 11: Nonlocal parameters.

\begin{tabular}{|c|c|c|c|c|c|c|}
\hline References & CNT & BC & $l / d$ & $e_{0}$ & $e_{0} a(\mathrm{~nm})$ & $m=\left(e_{0} a\right)^{2}\left(\mathrm{~nm}^{2}\right)$ \\
\hline Zhang et al. [39] & SWCNT & & 5.2 & $0.54-0.89$ & & \\
\hline \multirow[t]{2}{*}{ Duan et al. [18] } & $\operatorname{SWCNT}(5,5)$ & $\mathrm{C}-\mathrm{C}$ & $5.26-15.05$ & $0-4$ & & \\
\hline & & C-F & $5.26-15.05$ & $4-20$ & & \\
\hline \multirow[t]{2}{*}{ Zhang et al. [40] } & SWCNT $(5,5)$ & $\mathrm{C}-\mathrm{C}$ & $4.86-35.53$ & 1.25 & & \\
\hline & & $\mathrm{C}-\mathrm{F}$ & $4.67-35.34$ & 1.25 & & \\
\hline \multirow[t]{2}{*}{ Arash and Ansari [41] } & SWCNT $(5,5)$ & $\mathrm{C}-\mathrm{C}$ & $5.00-35.00$ & & 1.7 & \\
\hline & & C-F & $5.00-35.00$ & & 2.0 & \\
\hline \multirow[t]{4}{*}{ Ansari and Sahmani [20] } & $\operatorname{SWCNT}(8,8)(14,0)$ & S-S & $8.30-39.1$ & & & 0.19 \\
\hline & & $\mathrm{C}-\mathrm{C}$ & $8.30-39.1$ & & & 0.29 \\
\hline & & C-S & $8.30-39.1$ & & & 0.22 \\
\hline & & C-F & $8.30-39.1$ & & & 0.23 \\
\hline \multirow[t]{2}{*}{ This work } & $\operatorname{SWCNT}(5,5)$ & $\mathrm{C}-\mathrm{C}$ & $4.86-28.31$ & $4-16$ & $0.5-2.2$ & \\
\hline & & C-F & $4.67-28.07$ & $5-34$ & $0.8-4.9$ & \\
\hline \multirow[t]{3}{*}{ Ansari et al. [19] } & DWCNT $(5,5)(10,10)$ & S-S & $8.30-35.3$ & & 0.90 & \\
\hline & DWCNT $(9,0)(17,0)$ & $\mathrm{C}-\mathrm{C}$ & $8.30-35.3$ & & $0.87-0.88$ & \\
\hline & & C-S & $8.30-35.3$ & & 0.92 & \\
\hline \multirow[t]{3}{*}{ This work } & DWCNT $(5,5)(10,10)$ & S-S & $8.30-35.3$ & $0-75$ & $0-10.6$ & \\
\hline & & $C-C$ & $8.30-35.3$ & $0-69$ & $0-9.8$ & \\
\hline & & C-S & $8.30-35.3$ & $0-70$ & $0-10$ & \\
\hline
\end{tabular}




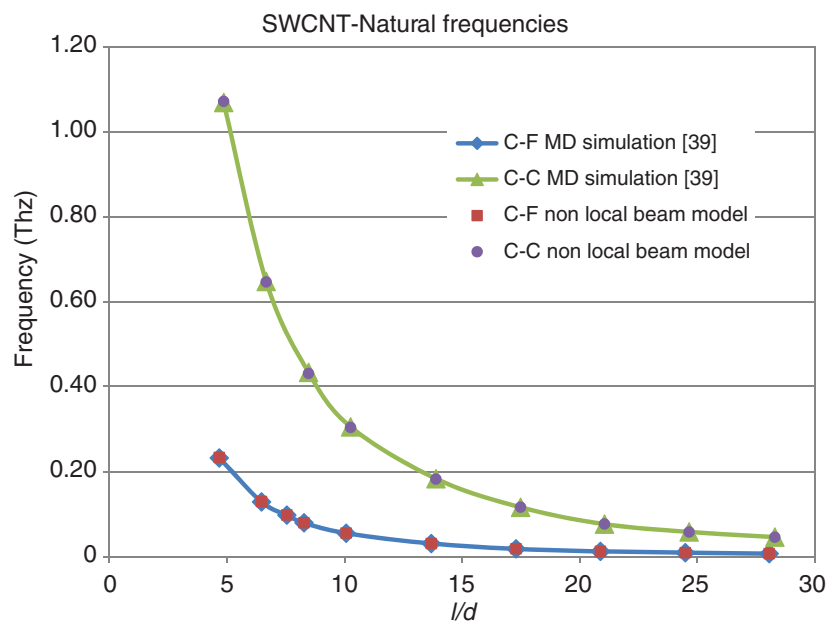

Figure 3: SWCNT natural frequencies from MD and nonlocal beam model.

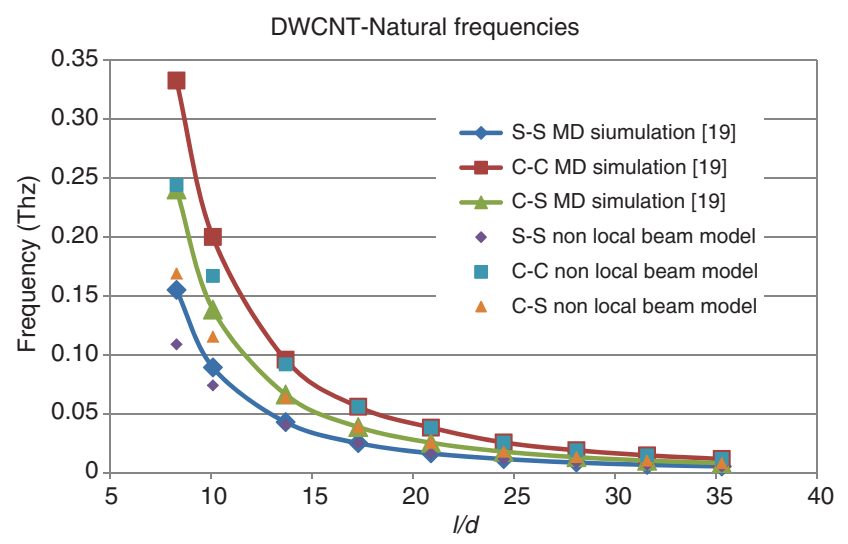

Figure 4: DWCNT natural frequencies from MD and nonlocal beam model.

\section{Conclusions}

A finite beam element on a HBM is proposed to study the transverse vibrations of DWCNTs, including those that could have initial deformations. The element mass and stiffness matrices are presented and can be programmed in any computational frame. The model is applicable to arbitrary boundary conditions. It was numerically validated against other results from the literature and the numerical results are in good agreement with those of referenced work.

A numerical study was carried out to study the influence of the nonlocal parameter on the coaxial and noncoaxial natural frequencies of different aspect ratios of DWCNT of different boundary conditions. The frequencies are very sensitive to the nonlocal parameter chosen for all boundary conditions, aspect ratio, and both TBM and EBM. The coaxial frequencies decrease, whereas the nonlocal parameter increases, and this influence is significant in higher modes. An opposite behavior is observed in noncoaxial frequencies, but the increase in the frequency is minor and can be neglected. The nonlocal parameter is calibrated for SWCNT and DWCNT. It depends on the beam aspect ratio and boundary conditions.

Acknowledgments: The financial support of the CONICET and the Universidad Nacional de Cuyo is gratefully acknowledged.

\section{References}

[1] Nakabayashi D, Moreau ALD, Coluci VR, Galvão DS, Cotta MA, Ugarte D. Nano Lett. 2008, 8, 842-847.

[2] Coleman JN, Khan U, Blau WJ, Gun'ko YK. Carbon 2006, 44, 1624-1652.

[3] Murshed SMS, Nieto de Castro CA. Renew. Sustain. Energy Rev. 2014, 37, 155-167.

[4] Kruss S, Hilmer A, Zhang J, Reuel N, Mu B, Strano M. Adv. Drug Deliv. Rev. 2013, 65, 1933-1950.

[5] Hu YG, Liew KM, Wang Q. Proc. Eng. 2012, 31, 343-347.

[6] Ansari R, Sadeghi F, Ajori S. Mech. Res. Commun. 2013, 47, 18-23.

[7] Ranjbartoreh A, Wang G. Physica E 2010, 43, 202-206.

[8] Li C, Chou TW. Appl. Phys. Lett. 2004, 84, 5246-5248.

[9] Avila AF, Eduardo AC, Neto AS. Comput. Struct. 2011, 89, 878-892.

[10] Merli R, Lazaro C, Monleon S, Domingo A. Comput. Struct. 2013, 127, 68-87.

[11] Ambrosini D, de Borbón F. Comput. Mater. Sci. 2012, 53 , 214-218.

[12] Liew KM, Wang Q. Int. J. Eng. Sci. 2007, 45, 227-241.

[13] Lei X, Natsuki T, Shi J, Ni Q. Phys. Lett. A 2011, 375, 2416-2421.

[14] Arash B, Wang Q. Comput. Mater. Sci. 2012, 51, 303-313.

[15] Eringen AC. Nonlocal Continuum Field Theories. SpringerVerlag New York, Inc.: New York, 2002.

[16] Peddieson J, Buchanan G, McNitt RP. Int. J. Eng. Sci. 2003, 41, 305-312.

[17] Wang Q.J. Appl. Phys. 2005, 98, 124301.

[18] Duan W, Wang C, Zhang Y. J. Appl. Phys. 2007, 101, 024305.

[19] Ansari R, Rouhi H, Sahmani S. Int. J. Mech. Sci. 2011, 53, 786-792.

[20] Ansari R, Sahmani S. Commun. Nonlinear Sci. Numer. Simul. 2012, 17, 1965-1979.

[21] Reddy JN. Int. J. Eng. Sci. 2007, 45, 288-307.

[22] Lu P, Lee HP, Lu C, Zhang PQ. Int. J. Solids Struct. 2007, 44, 5289-5300.

[23] Di Paola M, Failla G, Sofi A, Zingales M. Int. J. Mech. Sci. 2011, 53, 676-687. 
[24] Xu KY, Aifantis EC, Yan YH. J. Appl. Mech. ASME 2008, 75, 210131-210139.

[25] Xu KY, Alnefaie KA, Abu-Hamdeh NH, Almitani KH, Aifantis EC. Acta Mech. Solida Sin. 2014, 27, 345-352.

[26] Adali S. Nano Lett. 2009, 9, 1737-1741.

[27] Shakouri A, Lin RM, Ng TY. J. Appl. Phys. 2009, 106, 094307.

[28] Eltaher MA, Alshorbagy AE, Mahmoud FF. Appl. Math. Modell. 2013, 37, 4787-4797.

[29] Alotta G, Failla G, Zingales M. Finite Elem. Anal. Des. 2014, 89, 77-92.

[30] Ambrosini D. Thin Wall Struct. 2009, 47, 629-636.

[31] Ru C. Phys. Rev. B 2000, 62, 16962-16967.

[32] Saito R, Matsuo R, Kimura T, Dresselhaus G, Dresselhaus M. Chem. Phys. Lett. 2001, 348, 187-193.
[33] de Borbón F, Ambrosini D. Comput. Mater. Sci. 2012, 65, 504-508.

[34] Becker EB, Carey GF, Oden JT. Finite Elements: An Introduction, Vol 1. Prentice Hall: New Jersey, 1981.

[35] Zienkiewicz O, Taylor R. The Finite Element Method. 4th ed. McGraw-Hill: New York, 1994.

[36] de Borbón F, Mirasso A, Ambrosini D. Comput. Struct. 2011, 89 , 1406-1416.

[37] Clough R, Penzien J. Dynamics of Structures. McGraw-Hill: Singapore, 1975.

[38] Ehteshami H, Hajabasi M. Physica E 2011, 44, 270-285.

[39] Zhang YY, Tan VBC, Wang CM. J. Appl. Phys. 2006, 100, 074304.

[40] Zhang YY, Wang CM, Tan VBC. Adv. Appl. Math. Mech. 2009, 1, 89-106.

[41] Arash B, Ansari R. Physica E 2010, 42, 2058-2064. 